\title{
Design of Web Ranking Module using Genetic Algorithm
}

\author{
Vikas Thada \\ Research Scholar \\ Dr.K.N.M. University \\ Newai, India
}

\author{
Vivek Jaglan, Ph.D \\ Asst.Prof(CSE),ASET \\ Amity University \\ Gurgaon, India
}

\begin{abstract}
Crawling is a process in which web search engines collect data from the web. Focused crawling is a special type of crawling process where crawler look for information related to a predefined topic[1].In this paper a method for finding out the most relevant document among a set of documents for the given set of keyword is presented. Relevance checking is done with the help of Rogers-Tanimoto, MountFord and BaroniUrbani/Buser similarity coefficients. The method uses genetic algorithm to show that the average similarity of documents to the query increases when Probability of mutation is taken as low and Probability of crossover is taken as high. The method does the performance analysis of different similarity coefficients on the same set of documents and applies ranking to the documents whose relevancy is highest among the three coefficients.
\end{abstract}

\section{General Terms}

Growth, retrieval, crawl, engine

\section{Keywords}

Relevancy, similarity, coefficients, genetic

\section{INTRODUCTION}

Design of most focused crawlers is based on the vector space model. The model is used to judge the evenness of web pages and general web search algorithms. The relevance in turn work as guide in following target links [2]. One of the most important module of search engine is ranking module. The task of ranking module is to assign some ranking score to relevant pages using some criterion. Output of ranking module is an ordered set of pages according to their rank i.e. pages with high rank are near the top of the list and low rank pages are at the bottom of the list. These pages are then presented to the user in their ranking order. A GA based approach using Rogers-Tanimoto, MountFord and BaroniUrbani/Buser similarity coefficients is taken in this paper for ranking the retrieved documents.

\section{GENETIC ALGORITHM}

GAs are search algorithms that follow the concept of natural selection and genetics [3]. GA are powerful and very efficient search and optimization techniques motivated by the natural selection theory of Darwin [4].

Genetic Algorithms [5] are based on the principle of heredity and evolution which claims "in each generation the stronger individual survives and the weaker dies". Therefore, each new generation would contain stronger (fitter) individuals in contrast to its ancestors. The process of GA's is iteration based of constant population size of candidate solutions. In each generation/iteration each chromosome's fitness in the current population is evaluated and new population evolves. Chromosomes with higher fitness values goes through reproduction phase in which selection, crossover and mutation operators are applied to get new population. Chromosomes with lower fitness values are discarded. Again this generated new population is evaluated and selection, crossover, mutation operators are applied. This process continues until we get an optimal solution for the given problem

\subsection{Fitness Evaluation}

Fitness function is a function which is responsible for evaluating some value to indicate among number of solutions which one is optimum. It can also be considered as a measure of performance or fitness to show how fit is the candidate solution. The problem of IRS using GA is to retrieve documents using this fitness function. For finding the relevant document on the basis of some similarity measures we can have number of relevancy methods.

Table 1: Coefficients Used As Fitness Function In Research [6]

\begin{tabular}{|l|l|l|}
\hline S.N & Cofficient Name & Similarity Formula \\
\hline 1. & $\begin{array}{l}\text { Rogers \& } \\
\text { Tanimoto }\end{array}$ & $(\mathrm{p}+\mathrm{s}) /(\mathrm{p}+2 *(\mathrm{q}+\mathrm{r})+\mathrm{s})$ \\
\hline $\mathbf{2 .}$ & $\begin{array}{l}\text { Baroni- } \\
\text { Urbani/Buser }\end{array}$ & $\begin{array}{l}(\mathrm{p}+\mathrm{sqrt}(\mathrm{p} * \mathrm{~s})) / \\
(\mathrm{p}+\mathrm{q}+\mathrm{r}+\mathrm{sqrt}(\mathrm{p} * \mathrm{~s}))\end{array}$ \\
\hline 3. & Mountford & $2 * \mathrm{p} /(2 * \mathrm{q} * \mathrm{r}+\mathrm{p} * \mathrm{q}+\mathrm{p} * \mathrm{r}))$ \\
\hline
\end{tabular}

For the calculation of similarity metric we define few parameters $\mathrm{p}, \mathrm{q}, \mathrm{r}$ and $\mathrm{s}$ as $(\mathrm{n}=\mathrm{p}+\mathrm{q}+\mathrm{r}+\mathrm{s})$.

$\mathrm{p}=(\mathrm{x}=1$ and $\mathrm{y}=1)$ (total match)

$\mathrm{q}=(\mathrm{x}=1$ and $\mathrm{y}=0)($ single match $)$

$\mathrm{r}=(\mathrm{x}=0$ and $\mathrm{y}=1)$ (single match)

$\mathrm{s}=(\mathrm{x}=0$ and $\mathrm{y}=0)$ (no match)

This is shown in table 2 , where

Table 2: Variables Used to Calculate Binary Similarities/Dissimilarities[7,6,8]

\begin{tabular}{|l|l|l|}
\hline$x=1$ & $\begin{array}{l}\mathrm{p}=1 / 1 \text { in both } \\
\text { A and } B\end{array}$ & $\mathrm{q}=1 / 0$ only in A \\
\hline $\mathrm{x}=0$ & $\begin{array}{l}\mathrm{r}=0 / 1 \text { only in } \\
\text { B }\end{array}$ & $\begin{array}{l}\mathrm{s}=0 / 0 \text { in none of } \\
\text { A and } \mathrm{B}\end{array}$ \\
\hline
\end{tabular}

Where A and B may be any query or document represented in binary form. 


\subsection{Selection}

Once the fitness evaluation process is done next step is to perform selection operation. Process of selection operation is based on the principle of "survival of the fittest'. Higher fitness valued chromosomes goes through reproduction. Lower fitness valued chromosomes are discarded. There are number of ways to implement this operator, but all relies on the concept that candidates with good fitness values are to be preferred over poor fitness values. The idea is to give preference to better individuals. This selection operation does the replication of candidate chromosomes with good fitness values and eliminating those with poor fitness values [9].

\begin{tabular}{|l|l|}
\hline Individual & Fitness \\
\hline $\mathrm{A}$ & 8 \\
\hline $\mathrm{B}$ & 7 \\
\hline $\mathrm{C}$ & 3 \\
\hline $\mathrm{D}$ & 5 \\
\hline
\end{tabular}$\quad$\begin{tabular}{|l|l|}
\hline Individual & Fitness \\
\hline $\mathrm{A}$ & 8 \\
\hline $\mathrm{B}$ & 7 \\
\hline $\mathrm{A}$ & 8 \\
\hline $\mathrm{D}$ & 5 \\
\hline
\end{tabular}

Fig 1: Selection Operator on a Population of 4 Individuals[9]

The research work uses roulette wheel selection method as selection operator [10]. It is also known as fitness proportionate selection method.

\subsection{Crossover[3,11]}

In the crossover operation mating of two chromosomes is performed that gives birth to two new offspring. This operation of crossover always happens with one parameter that is known as probability of crossover (ProC). When ProC is say 0.8 it means only $80 \%$ of the total population goes for crossover operation. Rests $20 \%$ chromosomes remain abstain from this operation and has no effect of crossover. Motive behind performing crossover operation is to explore new solutions and exploit use of old solutions. GA forms an optimum solution by mating two fit chromosomes together. Chromosomes with higher fitness will always have good selection probability then others with lower fitness values, thus a good solution moves from one generation to next generation

\section{One point crossover (crossover point 7 )}

\begin{tabular}{|l|l|l|l|l|l|l|l|l|l|l|l|l|l|l|l|l|l|l|l|l|l|}
\hline Doc1 & $\mathbf{1}$ & $\mathbf{0}$ & $\mathbf{1}$ & $\mathbf{0}$ & $\mathbf{0}$ & $\mathbf{0}$ & $\mathbf{1}$ & 0 & 0 & 0 & 0 & 1 & 0 & 0 & 0 & 0 & 0 & 1 & 0 & 0 & 0 \\
\hline Doc2 & 0 & 0 & 1 & 0 & 0 & 0 & 1 & $\mathbf{0}$ & $\mathbf{1}$ & $\mathbf{0}$ & $\mathbf{0}$ & $\mathbf{1}$ & $\mathbf{0}$ & $\mathbf{0}$ & $\mathbf{0}$ & $\mathbf{0}$ & $\mathbf{0}$ & $\mathbf{0}$ & $\mathbf{0}$ & $\mathbf{0}$ & $\mathbf{1}$ \\
\hline
\end{tabular}

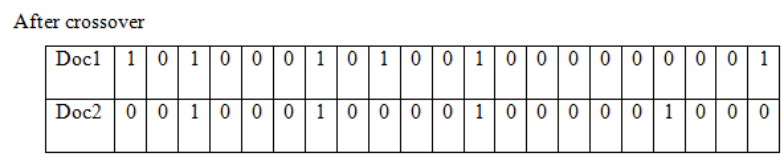

\subsection{Mutation[3,11]}

Mutation involves changing one bit of a chromosome from 0 to 1 or viceversa. This is performed under the constraint parameter called probability of mutation (ProM). For example if ProM is 0.10 then $10 \%$ genes of total chromosomes will go for mutation. The concept of mutation is based on this natural theory that varying breeds are possible only by varying gene values. After this operation fitness quality of new chromosomes may be high or low then old ones. In case new chromosomes are poor then old ones they are removed during selection process. The motive behind mutation is regaining the lost and discovering varying breeds. For example: randomly mutate chromosome at position 5

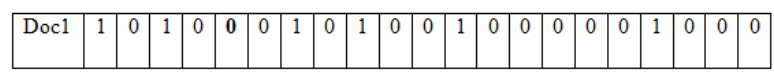

After mutation (bit number 5 is mutated i.e. bit is negated)

\begin{tabular}{|l|l|l|l|l|l|l|l|l|l|l|l|l|l|l|l|l|l|l|l|l|l|}
\hline Doc1 & 0 & 0 & 1 & 0 & $\mathbf{l}$ & 0 & 1 & 0 & 0 & 0 & 0 & 1 & 0 & 0 & 0 & 0 & 0 & 0 & 0 & 0 & 1 \\
\hline
\end{tabular}

Fig 3: Mutation Operation Explained

\section{RESEARCH TOOLS}

This section is about the introduction of tools which helped us to find the top keywords from relevant document. These keywords actually used for making the chromosomes; backbone of GA in which the research implementation work was carried out. We discuss a brief introduction of tools Text Analyzer and Keyword Density Checker. Both the tools were used to find out top most keywords present on webpage with highest frequency.

\subsubsection{Textalyser}

Textalyser[12] is tool for analyzing text or website online. The tool can be used for calculating detailed statistics of text like frequency of top occurring words in text or webpage etc. The tool has good application for translation purpose and webmasters for ranking webpages, for simply normal users to know about top keywords from a text. Using this tool keyword density can be easily found out. Further relative importance of word or expressions can also be found out. Webmasters can use this tool for the analysis of the links on their pages.

\subsubsection{Keyword Density Checker}

The keyword density checker tool [13] is an online tool which can be used for finding density of top occurred keywords. These keywords are then displayed at the top. Input to the tool can be URL only against both URL and text for textalyser tool. The tool crawls the given URL, bring out words normally as done by search engine, remove punctuations and return result in the form of density of the top URL keywords in the form of keyword cloud.

Fig 2: Single Point Crossover Explained 


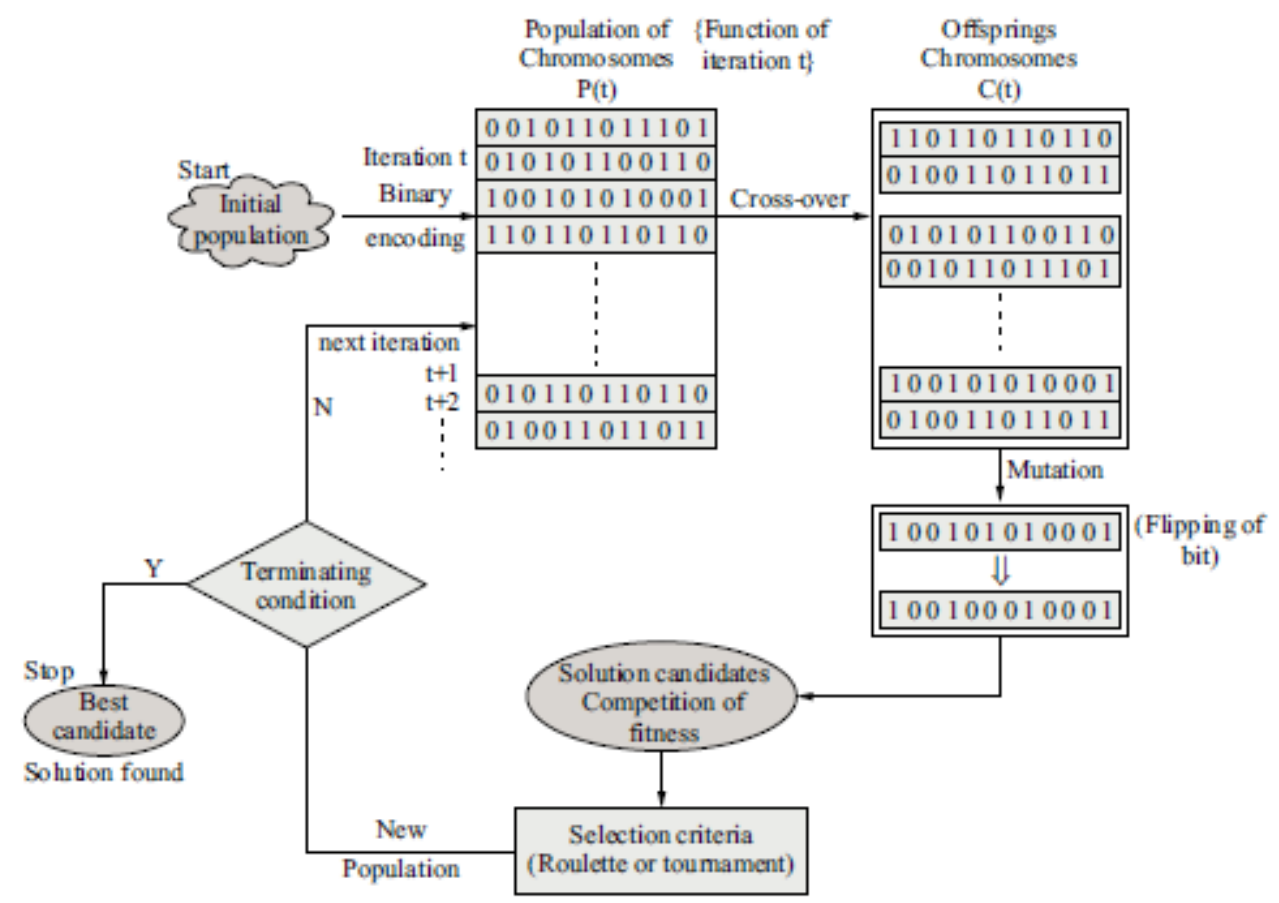

Fig 4: Basic Operation of Genetic Algorithm[14]

\section{EXPERIMENTAL SETUP \& RESULTS}

In general the web documents are encoded in strings of 0 's and 1's as shown in the figure 1. The documents have been obtained using some search query. The set of documents also contains some non relevant documents too. In the experimental setup of GA 50 documents have been retrieved for each different query. This set of 50 documents serves as document database. The query is also encoded in terms of string of 0 and 1. Process of experiment is as follows:

i. Query is input to the Google search engine.

ii. Top frequent keywords of each retrieved document are extracted using Textalyser or Keyword Density Checker online tool as discussed in chapter 3 and making list of keywords.

iii. Generate initial population by encoding retrieved documents to chromosomes for each query.

iv. Initial population now consists of these encoded documents. Pass this initial population to GA algorithm.

v. Either some maximum generation or some predefined fitness value is not achieved repeat step 4. At the end of this step we get an optimized document for retrieval.

vi. Optimized document chromosome can be decoded and document can be retrieved from stored documents related to each query.

vii. Rank the documents on the basis of fitness value as obtained in step 4.

\subsection{Experimentation}

The research work conducted the tests for 15 different queries and 30 documents for each query. The experimentation tests for all 15 queries with RT, MF and BUB coefficients chosen as fitness function. A complete MATLAB code has been written with roulette wheel selection operator, random point crossover with different rates of crossover and mutation. Experiment conducted with various GA parameters as : probability of crossover $\mathrm{ProC}=0.7,0.8$ and 0.9 along with probability of mutation (ProM $=0.01,0.10,0.30$ ) to do performance analysis of this GA based retrieval system. The efficiency parameter is average relevance. Average relevance from three fitness functions are compared and best combination for crossover and mutation probability for any one among three similarity function is selected as best . Using this best selected similarity function the document's rank before and after the algorithm are checked and new ranks to document are assigned. The retrieval process now retrieves documents using this new ranking scheme.

\section{Table 3: Queries Used in Research Work}

\begin{tabular}{|l|l|l|}
\hline $\begin{array}{l}\text { S. } \\
\text { N }\end{array}$ & $\begin{array}{l}\text { Chromosome } \\
\text { Length }\end{array}$ & Keywords \\
\hline 1. & 33 & Delhi, Gang, Rape, Case \\
\hline 2. & 39 & Bomb, Blast, Boston, Marathon \\
\hline 3. & 37 & Search, Engine, Optimization,Seo \\
\hline 4. & 49 & Osama, Hazare, Anti, Corruption \\
\hline 5. & 53 & Stock, Market, Mutual, Fund \\
\hline 6. & 47 & Fiber, Optic, Information, Technology \\
\hline 7. & 55 & Health, Medicine, Medical,Disease \\
\hline 8. & 66 & $\begin{array}{l}\text { Artificial,Intelligence,Neural, } \\
\text { Network }\end{array}$ \\
\hline 9. & 44 &
\end{tabular}




\begin{tabular}{|c|l|l|}
\hline 10. & 40 & Rajasthan, Royals, Amity,University \\
\hline 11. & 49 & Remote, Method, Invocation, RMI \\
\hline 12. & 61 & Gang, Rape, Kangaroo, Court \\
\hline 13. & 69 & Aam, Aadmi, Party, Arvind, Kejriwal \\
\hline 14. & 50 & $\begin{array}{l}\text { Genetic,Algorithms, Optimization, } \\
\text { Techniques }\end{array}$ \\
\hline 15. & 64 & Search, Engine, Web, Crawler \\
\hline
\end{tabular}

\subsection{Results}

In this section we show the results in tabular and graphical form for all three similarity coefficients viz. RT, MF and BUB. We vary probability of mutation ProM from 0.01 to 0.10 and 0.30 and keep $\mathrm{ProC}=0.5,0.7$ and 0.9 for all the values of ProM. Keeping together ProC and ProM we get total nine possible combinations for checking the performance of said similarity coefficients on retrieved documents. Out of nine possible results we got best was for $\mathrm{ProC}=0.9$ and ProM=0.1. Tables and figures for rest eight combinations have not been shown.

Table 4: Maximum Average Relevancy with $\mathrm{ProC}=0.9$ and ProM $=0.01$

\begin{tabular}{|c|c|c|c|}
\hline & \multicolumn{2}{|c|}{ ProC $=0.9$} & \multirow{2}{*}{$\begin{array}{r}\text { ProM=0.01 } \\
\text { BUB }\end{array}$} \\
\hline & RT & MF & \\
\hline Query1 & 0.6309 & 0.2948 & 0.6141 \\
\hline Query2 & 0.5442 & 0.2307 & 0.5446 \\
\hline Query3 & 0.5729 & 0.1871 & 0.5462 \\
\hline Query4 & 0.5541 & 0.1503 & 0.4695 \\
\hline Query5 & 0.5381 & 0.1258 & 0.4509 \\
\hline Query6 & 0.5348 & 0.1337 & 0.4702 \\
\hline Query7 & 0.5462 & 0.1182 & 0.4507 \\
\hline Query8 & 0.5268 & 0.088 & 0.4113 \\
\hline Query9 & 0.5852 & 0.1666 & 0.5198 \\
\hline Query10 & 0.5838 & 0.2001 & 0.5171 \\
\hline Query11 & 0.5604 & 0.1356 & 0.4397 \\
\hline Query12 & 0.5294 & 0.0848 & 0.4641 \\
\hline Query13 & 0.5345 & 0.0815 & 0.4241 \\
\hline Query14 & 0.5552 & 0.1457 & 0.4808 \\
\hline Query15 & 0.4987 & 0.088 & 0.4518 \\
\hline
\end{tabular}

Further it was observed that best average maximum fitness is given by Rogers-Tanimoto coefficient. Figure plotted using the above table is shown in Fig 4

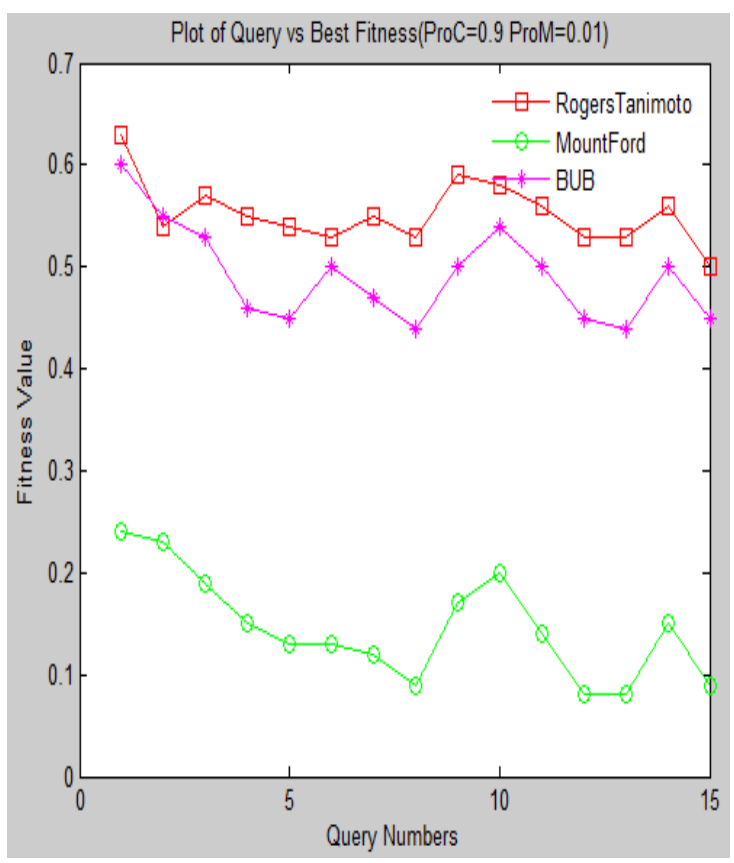

Fig 5: Query vs best fitness for $\mathrm{ProC}=0.9, \mathrm{ProM}=0.01$ using RT,MF and BUB

\subsection{Ranking of Documents}

To rank the documents using GA on the basis of fitness obtained using RT for the above parameters of GA for all queries, we run the MATLAB code for a fixed number of generations for RT and obtain the fitness values for each documents. The documents are ranked on the basis of this obtained fitness values. We show this only for first query Q1 $=\{$ Delhi, Gang, Rape, Case $\}$ in table . The hyperlinks for each of the documents are not shown in fact we name them as Di (i=1 to 30$)$.

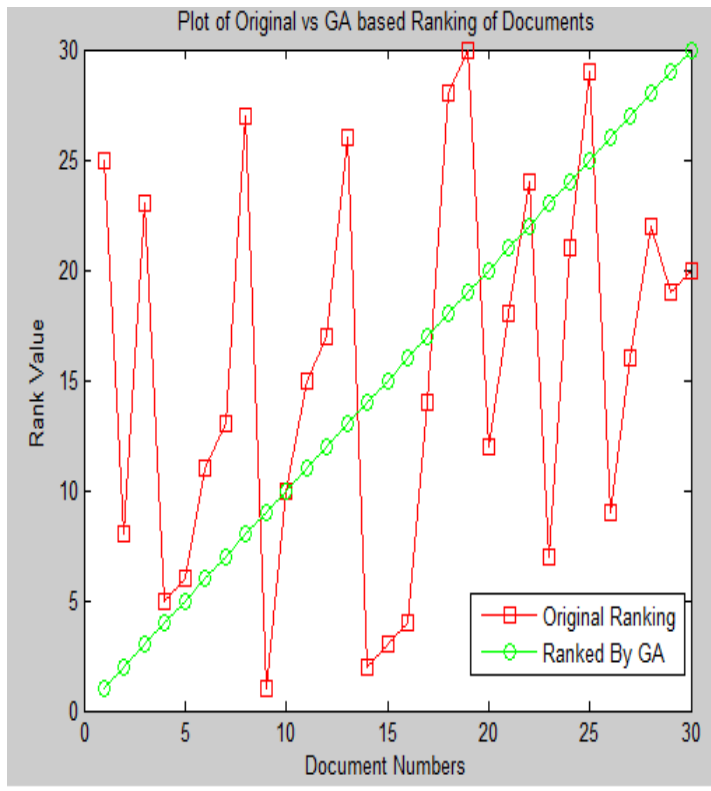

Fig 6: Comparative ranking of Original and GA based using RT similarity coefficient 
Table 5: New and Old Ranking of Documents Using GA and \% increase /Decrease

\begin{tabular}{|l|l|l|l|}
\hline Docs & $\begin{array}{l}\text { Original } \\
\text { Ranking }\end{array}$ & $\begin{array}{l}\text { New } \\
\text { Ranking }\end{array}$ & $\begin{array}{l}\text { \%Increase } \\
\text { / Decrease } \\
\text { in Ranking }\end{array}$ \\
\hline Doc 1 & 25 & 1 & 80 \\
\hline Doc 2 & 8 & 2 & 20 \\
\hline Doc 3 & 23 & 3 & 66.67 \\
\hline Doc 4 & 5 & 4 & 3.33 \\
\hline Doc 5 & 6 & 5 & 3.33 \\
\hline Doc 6 & 11 & 6 & 16.67 \\
\hline Doc 7 & 13 & 7 & 20 \\
\hline Doc 8 & 27 & 8 & 63.33 \\
\hline Doc 9 & 1 & 9 & -26.67 \\
\hline Doc 10 & 10 & 10 & 0 \\
\hline Doc 11 & 15 & 11 & 13.33 \\
\hline Doc 12 & 17 & 12 & 16.67 \\
\hline Doc 13 & 26 & 13 & 43.33 \\
\hline Doc 14 & 2 & 14 & -40 \\
\hline Doc 15 & 3 & 15 & -40 \\
\hline Doc 16 & 4 & 16 & -40 \\
\hline Doc 17 & 14 & 17 & -10 \\
\hline Doc 18 & 28 & 18 & 33.33 \\
\hline Doc 19 & 30 & 19 & 36.67 \\
\hline Doc 20 & 12 & 20 & -26.67 \\
\hline Doc 21 & 18 & 21 & -10 \\
\hline Doc 22 & 24 & 22 & 6.67 \\
\hline Doc 23 & 7 & 23 & -53.33 \\
\hline Doc 24 & 21 & 24 & -10 \\
\hline Doc 25 & 29 & 25 & 13.33 \\
\hline Doc 26 & 9 & 26 & -56.67 \\
\hline Doc 27 & 16 & 27 & -36.67 \\
\hline Doc 28 & 22 & 28 & -20 \\
\hline Doc 29 & 19 & 29 & -33.33 \\
\hline Doc 30 & 20 & 30 & -33.33 \\
\hline & & & \\
\hline
\end{tabular}

The comparison of old and new rankings and percentage improvement is shown graphically in the figures below:

The figure 4.23 and 4.24 shows that document having rank 25 has got new rank 1 and percentage improvement in rank is 80 . Similarly another document having old rank as 8 has moved to new rank 2 and got $20 \%$ increment in rank because of our applied GA with RT.

We have also checked online by opening pages with new rank and they were providing the good result as required by the corresponding query. Similar type of work was carried out for other queries and their related documents but as not to extend the length of this research paper we have provided ranking table and figure only for one query.

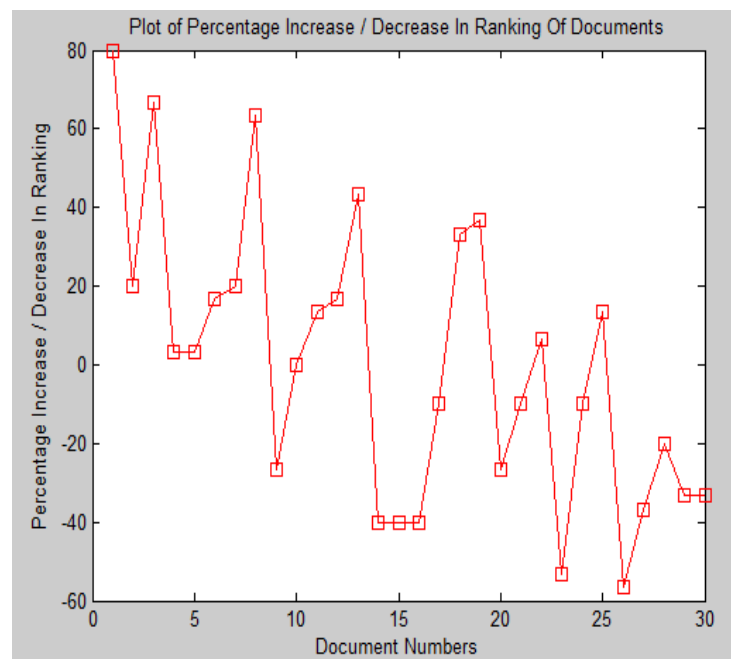

Fig 7: Percentage Increase/Decrease in Ranking of Documents

\section{CONCLUSION}

The research work has calculated average maximum fitness value using RT, BUB and MF similarity coefficients. In the work ProC and ProM was varied from 0.7-0.9 and \{0.01, $0.10,0.30\}$ respectively. It has been shown that average relevancy of retrieved document increases when PC is increased and ProM is decreased. Using the best average fitness value among RT, MF and BUB the research work applies new ranking to retrieved documents. The goal was to design the web ranking module using genetic algorithm and focused crawling and good results are a manifest to this research work. In this way searching has been made easy, so more relevant document or web pages can be retrieved easily. Results show that average relevance of document increased up to $63 \%$. In this way if focused crawler have key set with more relevancy then the retrieved data is more relevant for local collection of a search engine which improves the crawling performance.

\section{REFERENCES}

[1]. B. Novak "A Survey Of Focused Web Crawling Algorithms", Proceedings of SIKDD, pp. 55-58, 12-15 Oct 2004

[2]. http://www.wikipedia.org/Web_Crawler

[3] B.Klabbankoh, O.Pinngern. "applied genetic algorithms in information retrieval" Proceeding of IEEE ,pp.702711,Nov 2004

[4] S.S.Satya and P.Simon, "Review on Applicability of Genetic Algorithm to Web Search," International Journal of Computer Theory and Engineering, vol. 1, no. 4, pp. 450-455, 2009.

[5] Shokouhi, M.; Chubak, P.; Raeesy, Z " Enhancing focused crawling with genetic algorithms" Vol: 4-6, pp.503508,2005 .

[6]www.sequentix.de/gelquest/help/distance_measures.htm

[7] V.Consonni and R. Todeschini ,New Similarity Coefficients for Binary Data", Communications in Mathematical and in Computer Chemistry, pp.581-592, 2012 
[8] H.Wolda, "Similarity Indices, Sample Size and Diversity", OecoIogia-Springer-Verlag ,pp. 296-302,1981

[9] M.A.Kauser, M. Nasar, S.K.Singh, “A Detailed Study on Information Retrieval using Genetic Algorithm", Journal of Industrial and Intelligent Information vol. 1, no. 3, pp.122-127 Sep 2013.

[10]http://en.wikipedia/wiki/Fitness_Proportionate_Selection
[11] J.R. Koza, “ Survey Of Genetic Algorithms And Genetic Programming", Proceedings of the Wescon, pp.589595,1995

[12] http://textalyser.net/

[13]http://www.webconfs.com/keyword-density-checker.php.

[14] V.Thada, V.Jaglan, "Use of Genetic Algorithm in Web Information Retrieval", International Journal of Emerging Technologies in Computational and Applied Sciences, vol.7,no.3,pp.278-281, Feb,2014 